\title{
Pick-up Ion Scattering in the Outer Heliosheath - Implications for IBEX and Voyager 1 Observations
}

\author{
Jacek Niemiec* \\ Instytut Fizyki Jadrowej PAN, ul. Radzikowskiego 152, 31-342 Kraków, Poland \\ E-mail: Jacek.Niemiec@ifj.edu.pl \\ Vladimir Florinski, Jacob Heerikhuisen \\ Department of Space Science, University of Alabama in Huntsville, Huntsville, AL 35899, USA

\section{Ken-Ichi Nishikawa} \\ Department of Physics, University of Alabama in Huntsville, Huntsville, AL 35899, USA
}

\begin{abstract}
The nearly circular band of energetic neutral atom (ENA) emission dominating the field of view of the Interplanetary Boundary EXplorer satellite (IBEX), is most commonly attributed to the effect of charge exchange of secondary pick-up ions (PUIs) gyrating about the magnetic field in the outer heliosheath and the interstellar space beyond. The main difficulty with this model is the problem with stability of ring distributions that tend to generate waves and scatter onto a shell on time scales too short for charge exchange to occur. We performed a careful study of the ring instability using theoretical dispersion relations and fully kinetic PIC simulations that included electron dynamics. In addition to several prescribed distributions, we used primary ENA fluxes from the global kinetic-neutral model of the heliosphere to obtain a smooth empirical representation of the PUI ring produced from solar-wind and heliosheath ENAs. Simulations reveal that the ring distribution generates both left- and right-hand polarized waves traveling parallel to the background magnetic field. Narrow rings generate abundant magnetic fluctuations at the resonant frequencies, whereas simulations with broadened rings show very low levels of turbulence. Nonetheless, particle scattering occurs for every type of rings we studied. Results are discussed in the context of IBEX observations of the ribbon and the recent Voyager 1 in situ measurements of magnetic field fluctuations in the outer heliosheath.
\end{abstract}

The 34th International Cosmic Ray Conference,

30 July- 6 August, 2015

The Hague, The Netherlands

\footnotetext{
* Speaker.
} 


\section{Introduction}

NASA's Interstellar Boundary EXplorer (IBEX) spacecraft generates all-sky maps of the energetic neutral atom (ENA) flux. Its first maps showed an unexpected "ribbon" of enhanced flux that encircled the sky $[1,2,3,4]$, and subsequent maps have shown this feature to be present in the same location over many years. Since its discovery, the ribbon has attracted more than a dozen explanations (for a recent summary see [5]). The ribbon's energy characteristics and helio-lattitude dependence suggest a connection to the supersonic solar wind, which has made the so-called "secondary ENA" model one of the most favored. In this mechanism ENAs created in the supersonic solar wind, due to charge-exchange between an interstellar neutral and a solar wind proton, exit the heliosphere and charge-exchange in the outer heliosheath to form a population of pick-up ions (PUIs). These PUIs charge-exchange again to become "secondary" ENAs which form the ribbon.

A key step in the secondary ENA mechanism is the wave-particle scattering of PUIs in the outer heliosheath (OHS) before being re-neutralized. Three limiting cases have been investigated. Firstly, [6] assumed weak scattering of PUIs onto a so-called "partial shell" distribution [7]. This approach yields enhanced ENA flux from regions near $\mathbf{B} \cdot \hat{\mathbf{r}} \simeq 0$ due to the fact that the partial shells are assumed to be close to a ring distribution at these locations, and hence have a higher phasespace density than an isotropic shell of the same number density. In fact, the $\mathbf{B} \cdot \hat{\mathbf{r}} \simeq 0$ condition is common to all secondary ENA models and has also been applied heuristically as a ribbon proxy $[8,9]$. Since $\mathbf{B}$ here is the interstellar magnetic field that wraps around the heliopause, the IBEX ribbon can be used in conjunction with models to deduce the properties of this field $[10,11]$.

In contrast to the partial shell model described above, two other approaches have assumed either no scattering [12] or scattering to only the forward hemisphere [13]. Both approaches still expect enhanced flux near $\mathbf{B} \cdot \hat{\mathbf{r}} \simeq 0$, the former due to a decrease in parallel velocity through the conservation of magnetic moment, and the latter due to a "spatial retention" effect. So while the secondary ENA mechanism appears to be the most likely explanation for the IBEX ribbon, further work is required to understand the nature of the PUIs in the outer heliosheath that form the source population for ribbon ENAs.

Recently, Voyager 1 performed the first in situ measurement of magnetic fluctuations in the region of space beyond the heliopause [14]. The fluctuation energy was only $0.05 \%$ of the total energy in the magnetic field, suggesting that the outer heliosheath may be a nearly laminar environment. This observation appears to rule out the instability, which was expected to generate plentiful turbulent fluctuations in the relevant wavenumber range [15]. It also brings into question models that rely on high levels of turbulence to retain the ions near their pick-up locations.

A possible solution was proposed (earlier) in [16]. Broadening of the PUI ring distribution leads to a decrease in growth rate eventually crossing into the stable region. Ions that make up the ring have a spread in velocity from nonuniform conditions in their places of origin (solar wind and the inner heliosheath). In linear theory, a warm Gaussian ring becomes stable with a velocity spread as small as $2 \mathrm{~km} \mathrm{~s}^{-1}$. Unfortunately, [16] have not performed any numerical simulations of the plasma with a broadened ring PUI distribution. It is the purpose of this paper to investigate numerically the stability of a PUI ring using high fidelity PIC simulations. 


\section{Initial ring distributions}

We are primarily interested in a stability of a PUI ring with a zero mean parallel velocity (perpendicular pick-up). Ring particles represent only about $0.01 \%$ of the total ion population in the outer heliosheath, but each carries an energy of about 500 times that of a thermal proton. A narrow ring PUI distribution injected at $90^{\circ}$ is always unstable in linear theory by virtue of a resonant interaction with right-hand polarized waves [17]. Hybrid-kinetic plasma simulations featuring large $(\sim 25,000)$ particles per cell showed that the plasma develops magnetic fluctuations that scatter the initial ring toward an isotropic shell. In the model of [17] the scattering process took only a few days, which is much shorter than the timescale for charge exchange (years). [18] included gradual injection of PUIs, but their simulations also resulted in an instability once the threshold density of accumulating ions was exceeded. Both papers focused on narrow rings, although [17] also studied a wider partial shell that also turned out to be unstable.

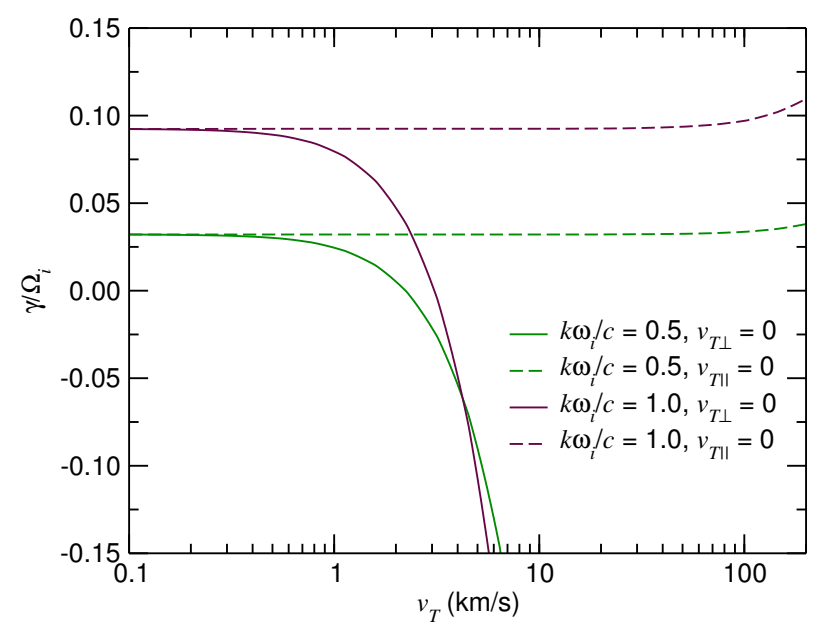

Figure 1: Theoretical linear growth rates, normalized to the ion cyclotron frequency, at $k=$ $0.5 c / \omega_{i}$ (green) and $k=c / \omega_{i}$ (dark red). Solid lines are for longitudinally broadened distributions; dashed lines are for flat rings with a spread in perpendicular velocity.

Here we investigate two different classes of distributions. The first is a warm toroidal distribution from [16] given by

$$
\begin{aligned}
f(\mathbf{v})=\frac{1}{\pi^{3 / 2} \delta v_{\perp}^{2} \delta v_{\|}}\left\{\exp \left(-\frac{v_{\perp, 0}^{2}}{\delta v_{\perp}^{2}}\right)+\frac{\sqrt{\pi} v_{\perp, 0}}{\delta v_{\perp}} \operatorname{erfc}\left(-\frac{v_{\perp, 0}}{\delta v_{\perp}}\right)\right\}^{-1} \\
\quad \times \exp \left[-\frac{\left(v_{\perp}-v_{\perp, 0}\right)^{2}}{\delta v_{\perp}^{2}}\right] \exp \left(-\frac{v_{\|}^{2}}{\delta v_{\|}^{2}}\right),
\end{aligned}
$$

where $v_{\perp, 0}$ is the major radius of the toroid and $\delta v_{\|}$and $\delta v_{\perp}$ are the thermal spreads parallel and perpendicular to the mean field, respectively. Figure 1 shows the theoretical linear growth rates for distribution (2.1) as a function of thermal spread for parallel wave propagation (the most unstable case). The growth rates were obtained by numerically solving the linear kinetic dispersion equation for a plasma with two populations of ions. Solid lines show growth rates for a ring widened in the parallel direction only, whereas dashed lines are for a ring spread in the direction normal to the field. Perpendicular spread has virtually no effect on the instability because it does not affect the resonance condition. Conversely, parallel broadening leads to a sharp drop in the growth rate and eventually, damping. A thermal speed of about $3 \mathrm{~km} \mathrm{~s}^{-1}$ is sufficient to suppress the instability, which is consistent with [16]. 
The second type of PUI distributions is derived from numerical simulations of the global heliosphere [11]. This model uses an MHD description for the background plasma and a Boltzmannkinetic description of the energetic neutral atoms that serve as parent populations of the ring PUIs. The three-dimensional ENA phase space density from the kinetic simulation is averaged over gyrophase angle to account for the gyrotropy of the pick-up ions. The complete PUI distribution consists of two populations: highly energetic ions produced from fast ENAs born in the solar wind (the so-called population 3, or "ring"), and diffuse ions produced from hot ENAs born in the inner heliosheath (population 2 or "halo"). Each distribution is fitted with a function

$$
f(\mathbf{v})=f_{0} e^{-\left(v-v_{0}\right)^{2} / \delta v^{2}} e^{-\mu^{2} / \delta \mu^{2}},
$$

where $\delta v$ and $\delta \mu$ are the widths of the distribution in total velocity and pitch angle, respectively, and $v_{0}$ is the radius of the ring. A typical fit is shown in Figure 2. The upturn near $v=0$ in the halo is most likely due to particles related to the interstellar neutral population; we ignore it here because these ions are essentially indistinguishable from the Maxwellian core.
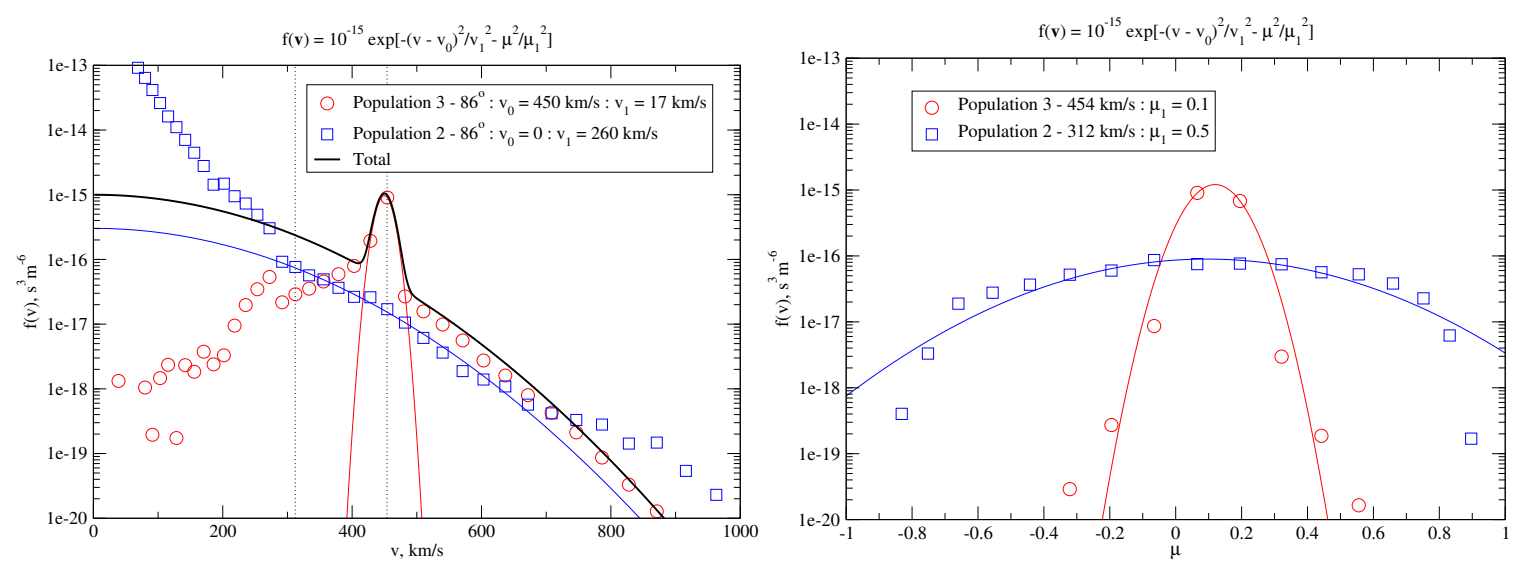

Figure 2: Fits (Eq. 2.2) to the distribution function of PUIs obtained from the global model of the helisphere. Left is velocity, right is pitch-angle.

\section{Particle-In-Cell Simulations}

\subsection{Numerical Particle-In-Cell model}

A two-dimensional PIC simulation of the long-time evolution of the PUI ring-beam instability is not computationally feasible for the realistic parameters used in the linear analysis. Therefore, parameters that approximate the physical conditions in the OHS region must be used instead. They have been selected based on extensive set of test runs. The main assumption was that any set of approximate parameters must preserve the scaling defined by the characteristic frequencies of the OHS plasma: $\omega_{\mathrm{pe}} \gg \omega_{\mathrm{pi}} \gg \Omega_{e} \gg \Omega_{i}$, where $\omega_{\mathrm{pe}}$ and $\omega_{\mathrm{pi}}$ are electron and ion plasma frequencies. With a reduced ion-to-electron mass ratio of $m_{i} / m_{e}=50$ assumed, a computationally feasible choice is the one with $\omega_{\mathrm{pe}} / \Omega_{e}=20, \omega_{\mathrm{pi}} / \Omega_{i} \simeq 141.42$, and thus $\omega_{\mathrm{pi}} / \Omega_{e} \simeq 2.83^{1}$. Assuming further

\footnotetext{
${ }^{1}$ For the realistic proton-electron mass ratio $m_{i} / m_{e}=1836$ and a dimensionless parameter $\omega_{\mathrm{pi}} / \Omega_{i}=c / v_{\mathrm{A}}=1.74 \times$ $10^{4}$ as in [17], these dependencies are numerically $\omega_{\mathrm{pe}} / \Omega_{e} \simeq 406$ and $\omega_{\mathrm{pi}} / \Omega_{e} \simeq 9.48$.
} 
the electron skindepth $\lambda_{\mathrm{se}}=8 \Delta$, where $\Delta$ is the cell size, and the electron thermal speed $v_{e, \text { th }}=$ $\sqrt{2 k T_{e} / m_{e}}=0.05 c$, one has the plasma beta $\beta=v_{i, \text { th }}^{2} / v_{\mathrm{A}}^{2}=1$, with the background ion thermal speed and the Alfvén velocity $v_{i, \text { th }}=v_{\mathrm{A}} \simeq 7.1 \times 10^{-3} c$. The time integration in the code can thus proceed with a time-step $\left(\delta t \Omega_{i}\right)^{-1}=16,000$. To stabilize the plasma against nonphysical effects on long times scales of the order of several tens of $\Omega_{i}^{-1}$ we apply a numerical PIC model used recently in [19]. The model takes advantage of second-order particle shapes (TSC - TriangularShaped Cloud) and uses a second-order FDTD field-solver with a weak Friedman filter [20] to suppress small-scale noise. We have checked that the system is satisfactorily stable if the number of (macro)particles per cell $N_{p p c}$ is above 250 .

For the parameters characterizing the PUI ring-beam we take the nonrelativistic solar wind speed $v_{\mathrm{SW}}=0.1 c \simeq 14 v_{\mathrm{A}}$ and choose a perpendicular pick-up angle $\alpha=90^{\circ}$. The ion ring-beam to background density ratio is $N_{\text {ring }} / N_{i}=0.025$. This has been chosen to increase the growth rate of plasma instabilities. The simulations have been performed on a spatial grid in the $x-y$ plane with periodic boundary conditions. Equal numbers of computational particles are used for all particle species - ring-beam ions and background electrons and ions. To match the desired $N_{\text {ring }} / N_{i}$ we apply the splitting method that assigns statistical weights to the ring beam ions and electrons, that accommodate also the excess population $\delta N_{e}=N_{e}-N_{\text {ring. }}$. Each ion particle is initialized at the same location as the corresponding electron, to ensure a vanishing initial charge density. The simulations were performed with a modified version of the TRISTAN code [21], which was adapted to work in 2D3V and parallelized using MPI [22].

\subsection{Results of PIC modeling}

The results of the PIC simulations of the ion ring-beam instability are presented here for four representative forms of the PUI distributions that have been investigated. Figure 3 shows the time evolution of the magnetic field energy density for the respective runs. All simulations use $N_{p p c}=$ 2500. By performing test runs with $N_{p p c}=250$, we have checked that for the selected number of particles per cell numerical effects do not modify the results.

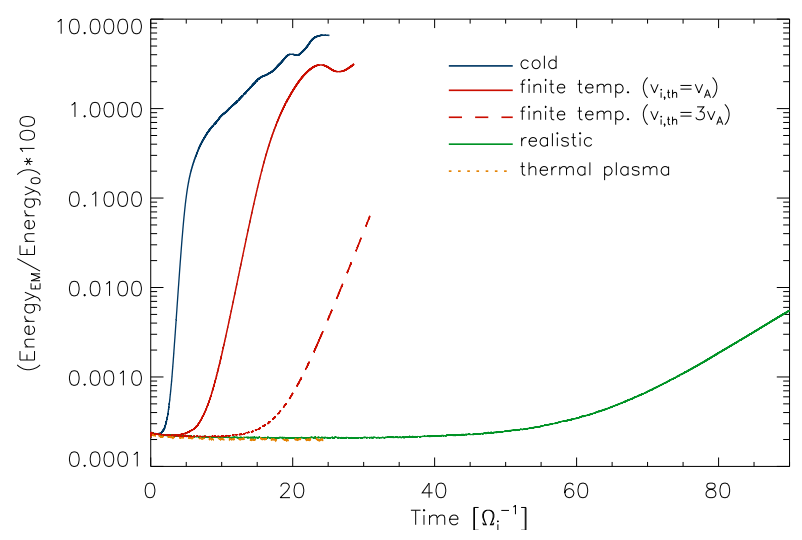

Figure 3: Temporal evolution of the magnetic field energy density for different ion-ring beam distributions (see text). Time is in units of the inverted ion cyclotron frequency.

The simplest case studied is the narrow PUI distribution, obtained in the $\delta v_{\perp}=\delta v_{\|}=0$ limit from Eq. 2.1 (Eq. 5 in [17]). It is shown in the velocity phase-space plot in Figure 4a. It will be referred here to as a cold ring distribution (blue line in Fig. 3). In this case a strong ion ring-beam instability occurs. It leads to the appearance of purely parallel magnetic modes that are right-hand 

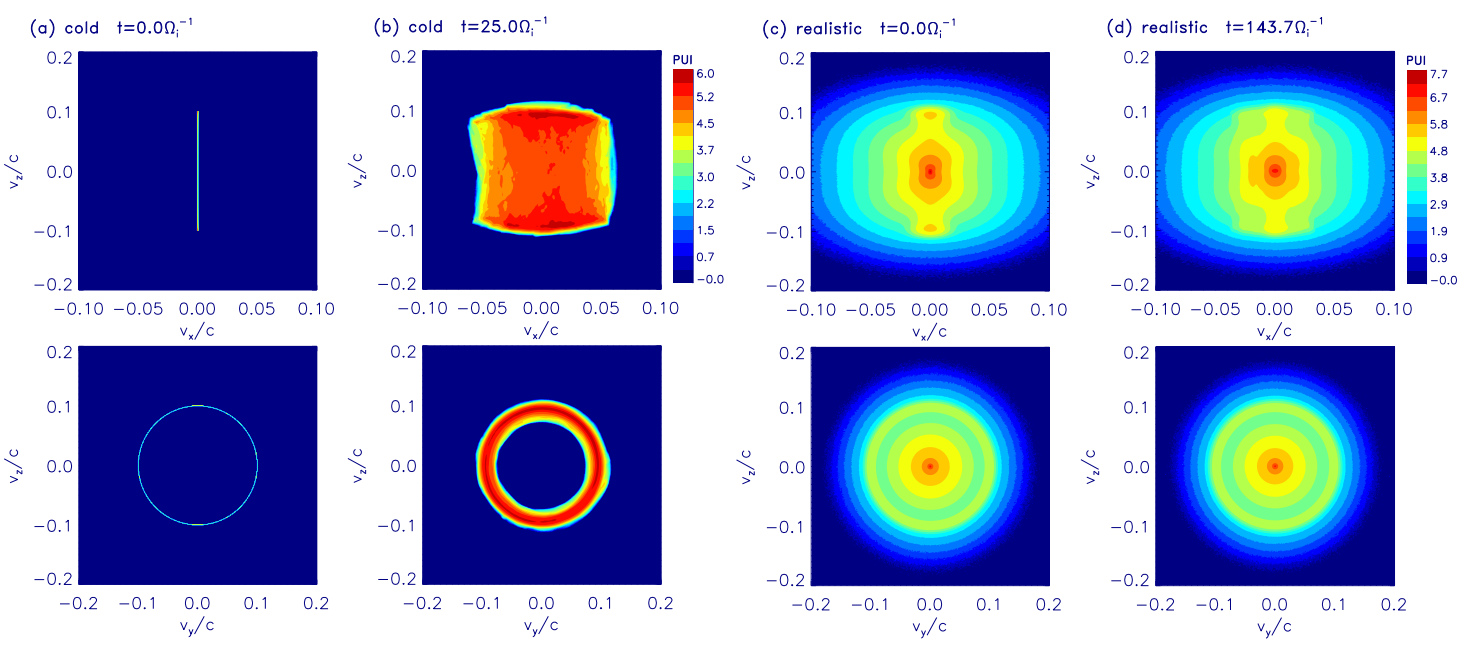

Figure 4: PUI velocity phase-space at $(a, c)$ the beginning and $(b, d)$ the end of the PIC simulation runs with (left) the cold and (right) the realistic ion ring distributions.
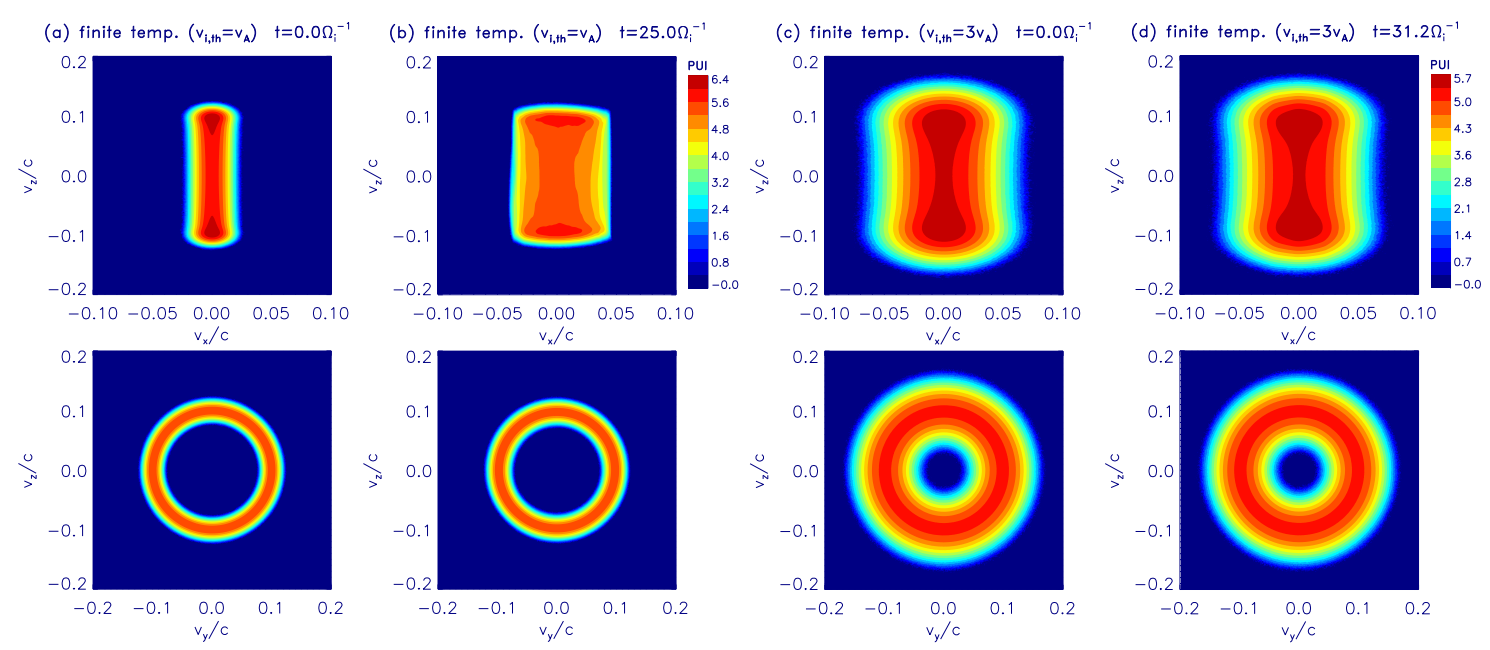

Figure 5: PUI velocity phase-space for finite temperature ion ring distributions (see Fig. 2).

circularly polarized $^{2}$ and are accompanied by strong parallel perturbations of the ring beam ion density. The amplitude and the wavelength of the modes grow in time. These characteristics are in agreement with the results of 1D hybrid modeling of [17], and we do not observe additional effects due to either an increased dimensionality of the system or the electron dynamics. As shown in Figure $4 \mathrm{~b}$, the ion ring becomes scattered in pitch angle to a nearly isotropic shell on a time-scale of several tens of ion cyclotron times.

The stability of the system is much improved for the PUI distribution derived from the realistic global model of the heliosphere (green line in Fig. 3, realistic distribution). For the density ratio of 6.3 between the halo and the ring distributions, we set their widths in total velocity to $v_{1}=0.58 v_{\mathrm{SW}}$

${ }^{2}$ For $\mathbf{B}_{0}=B_{0, \mathrm{x}} \hat{\mathbf{x}}$ and $\mathbf{k}=k_{x} \hat{\mathbf{x}}$, a plane electromagnetic wave of the form $\mathbf{B}_{1} \exp i(\mathbf{k} \cdot \mathbf{x}-\omega t), \mathbf{B}_{1}=B_{1, \mathrm{y}} \hat{\mathbf{y}}+B_{1, \mathrm{z}} \hat{\mathbf{z}}$ is right-hand circularly polarized if $B_{1, \mathrm{z}}=i B_{1, \mathrm{y}}$. Therefore, if viewed in the background plasma rest frame, the magnetic field vector of an approaching wave rotates anti-clockwise, in the direction of the electron gyromotion. 
and $v_{1}=v_{i, \text { th }}$, respectively (see Fig. 4c). The modes are strongly damped up to $t \sim 50 \Omega_{i}^{-1}$, when the instability emerges with a growth rate $\gamma \simeq 0.05 \Omega_{i}$ that is about 40 times slower than for the cold ring case. The instability growth continues until a near-saturation phase is reached at $t \sim 150 \Omega_{i}^{-1}$ (not shown in Fig. 3). By this time the distribution is only weakly scattered (see Fig. 4d).

The two other cases investigated concern finite temperature worm-toroidal distributions. Results for isotropic temperatures, given by $v_{\mathrm{Tb}}=\delta v_{\|}=\delta v_{\perp}=v_{i \text { th }}$ and $v_{\mathrm{Tb}}=3 v_{i, \text { th }}$ (Eq. 2.1 and Eq. 7 in [16], Fig. 5a and 5c) are presented in Figure 3 with red solid and dashed lines, respectively. One can see that neither beam temperature can stabilize the distribution since quite a strong growth of magnetic modes is observed in both cases. It is enough to scatter the colder ion ring onto a shell by $t \sim 30 \Omega_{i}^{-1}$ (Fig. 5b).

\subsection{Discussion}

The parameters in our PIC simulations were selected to facilitate a faster system evolution given limitations in available computing power. Direct application to the physical conditions in the OHS plasma is therefore not possible and some scaling needs to be performed. A possible way is to compare results of our PIC simulations to hybrid modeling discussed in [17]. In their simulation of the narrow beam that used realistic physical parameters, the ion ring was isotropized by $t \sim$ $4300 \Omega_{i}^{-1}$. If we assume that this corresponds to $t \sim 25 \Omega_{i}^{-1}$ in our simulation of the cold ring, we obtain a scaling factor of $\sim 170$. We can then apply this scaling to the results of our realistic run. By noting that little scattering occurs before the magnetic turbulence growth starts to saturate at $t \sim 120 \Omega_{i}^{-1}$, we can estimate that physical plasma with the realistic distribution is stable for at least $20,000 \Omega_{i}^{-1}$, i.e., about 10 days.

The above simple estimate might represent a lower limit for the ion ring stability time-scale. This is because of the unknown role of numerical scattering in a very-long duration run performed for the realistic distribution. In all other cases studied, the instability growth is fast and the noise effects do not influence the results. This argument can be further strengthened by noting that thermal plasma without ring ions is stable for the duration of the cold and finite temperature simulations (yellow dashed line in Fig. 3). However, if the emergence of the magnetic modes in the realistic simulation is due to the accumulated numerical noise, then increasing $N_{p p c}$ might further help in damping the turbulence.

\section{Summary}

Using PIC simulations, we have investigated the stability of two different classes of pick-up ion ring distribution that serve as parent ions of the ribbon ENAs observed by IBEX. Our results, performed with scaled versions of these distributions, show that the realistic broadened ring-plushalo distribution based on actual simulations of neutral atoms in the heliosphere, is the most stable, and exhibited virtually no scattering over $\sim 10$ days. This result suggests that such a distribution of ions may remain stable long enough for charge exchange to take place and generate a neutral atom that would be detected by IBEX. If this is the case, then the absence of magnetic fluctuations at Voyager 1 is naturally explained because the wave energy stays small (see Fig. 3). We caution, however, that Voyager 1 is not traveling in the direction of the ribbon, and the pick-up ions injected at its location have a finite pitch angle, whereas we only considered perpendicular pick-up. It is 
likely that the instability is not as severe at oblique pick-up angles, and is more readily suppressed by thermal broadening of the injected ring and the presence of the halo.

\section{Acknowledgments}

The work of J.N. is supported by Narodowe Centrum Nauki through research project DEC-2013/10/ E/ST9/00662. K.N. is supported by NSF AST-0908040, NNX12AH06G, NNX13AP-21G, and NNX13AP14G. V.F. was supported by NASA grant NNX12AH44G and by NSF grant AGS0955700. PIC simulations have been performed at the Pleiades facility at the NASA NAS.

\section{References}

[1] McComas, D. J., et al. 2009, Science, 326, 959

[2] Schwadron, N. A., et al. 2009, Science, 326, 966

[3] Funsten, H. O., et al. 2009, Science, 326, 964

[4] Fuselier, S. A., et al. 2009, Science, 326, 962

[5] McComas, D. J., Lewis, W. S., \& Schwadron, N. A. 2014, Rev. Geophys., 52, 118

[6] Heerikhuisen, J., et al. 2010a, ApJ, 708, L126

[7] Heerikhuisen, J., Pogorelov, N., \& Zank, G. 2010b, in American Institute of Physics Conference Series, Vol. 1302, American Institute of Physics Conference Series, ed. J. Le Roux, G. P. Zank, A. J. Coates, \& V. Florinski, 98

[8] Strumik, M., Ben-Jaffel, L., Ratkiewicz, R., \& Grygorczuk, J. 2011, ApJL, 741, L6

[9] Grygorczuk, J., Czechowski, A., \& Grzedzielski, S. 2014, ApJL, 789, L43

[10] Funsten, H. O., et al. 2013, ApJ, 776, 30

[11] Heerikhuisen, J., Zirnstein, E., Funsten, H., Pogorelov, N. V., \& Zank, G. P. 2014, ApJ, 784, 73

[12] Chalov, S. V., Alexashov, D. B., McComas, D., Izmodenov, V. V., Malama, Y. G., \& Schwadron, N. 2010, ApJ, 716, L99

[13] Schwadron, N. A., \& McComas, D. J. 2013, ApJ, 764, 92

[14] Burlaga, L. F., Florinski, V., and Ness, N. F. 2015, ApJL, 804, L31

[15] Burlaga, L. F., Ness, N. F., Florinski, V., \& Heerikhuisen, J. 2014, ApJ, 792, 134

[16] Summerlin, E.J., Vinas, A.F., Moore, T.E., Christian, E.R., \& Cooper, J.F., 2014, ApJ, 793, 93

[17] Florinski, V., Zank, G. P., Heerikhuisen, J., Hu, Q., \& Khazanov, I., 2010, ApJ, 719, 1097

[18] Liu, K., Moebius, E., Gary, S. P., and Winske, D. 2012, J. Geophys. Res., 117, A10102

[19] Niemiec, J., Pohl, M., Bret, A., \& Wieland, V. 2012, ApJ, 759, 73

[20] Greenwood, A. D., Cartwright, K. L., Luginslund, J. W., \& Baca, E.A., 2004, J. Comp. Phys., 201, 665

[21] Buneman, O. 1993, Computer Space Plasma Physics, ed. H. Matsumoto \& Y. Omura (Terra Scientific Publishing Company), 67-84

[22] Niemiec, J., Pohl, M., Stroman, T., \& Nishikawa, K.-I. 2008, ApJ, 684, 1174 